\title{
Aufkommen und Verteilung von Feststoffen in Kanalsystemen
}

\author{
Antje Welker
}

Eingegangen: 19. Juli 2010/Akzeptiert: 12. September 2010/Online veröffentlicht: 8. Oktober 2010

(C) Springer-Verlag 2010

Zusammenfassung Feststoffe spielen eine besondere Rolle bei der Bewertung von stofflichen Emissionen aus Kanalsystemen. Dies liegt vor allem daran, dass viele Schadstoffe mit Partikeln assoziiert in den Kanalsystemen transportiert und damit in die Oberflächengewässer eintragen werden. In diesem Beitrag werden alle wichtigen Emissionen aus den in Deutschland vorliegenden Kanalsystemen (Misch- und Trennsystem) genannt sowie typische Aufkommensbereiche abgeleitet. Des Weiteren werden die Daten von ausgewählten Schadstoffen (Blei, polyzyklische aromatische Kohlenwasserstoffe [PAK]) zusammengestellt und typische Aufkommensbereiche in den verschiedenen Abflussarten vorgestellt.

Aufbauend auf diesen Ergebnissen werden Schmutzfrachtberechnungen in einem fiktiven Einzugsgebiet vorgestellt. Schwerpunkt dieser Berechnungen ist der Vergleich der Emissionen aus Misch- und Trennsystemen, beispielhaft aufgezeigt für die Parameter „abfiltrierbare Stoffe“ (AFS), Blei und PAK.

Es ist zu erkennen, dass bei allen niederschlagsbürtigen Stoffen, beispielsweise dem Blei, die emittierte Gesamtfracht im Trennsystem wesentlich höher ist als die im Mischsystem. Dies ist durch die Mitbehandlung belasteter Niederschlagsabflüsse in Kläranlagen im Mischsystem begründet. Durch gute Rückhalteleistungen in der Kläranlage werden viele Stoffe in den Klärschlamm verfrachtet; hierdurch gelangen geringere Mengen in den Ablauf und damit in die Fließgewässer. Eine pauschale Bestätigung, dass Trennsysteme die besseren Kanalsysteme sind, kann nach diesen Ergebnissen nicht pauschal gegeben werden.

Verantwortliche Herausgeber: Organisationskomitee der Tagung

A. Welker $(\square)$

FH Frankfurt, FG Siedlungswasserwirtschaft,

Nibelungenplatz 1, 60318 Frankfurt am Main, Deutschland

E-Mail: antje.welker@fb1.fh-frankfurt.de
Insgesamt können durch den Vergleich eines Mischsystems und eines Trennsystems in einem fiktiven Beispieleinzugsgebiet Tendenzen zum Aufkommen und zur Verteilung herkömmlicher und möglicherweise in Zukunft bedeutsamer Stoffe mit analogen Stoffeigenschaften aufgezeigt werden.

Schlüsselwörter Feststoffe $\cdot$ Kanalsysteme $\cdot$ Schadstoffe $\cdot$ Schmutzfrachtberechnungen

\section{Occurrence and fate of solids in sewer systems}

Abstract Suspendend solids (SS) are important parameters in the assessment of potential effects of emissions out of sewer systems. This is due to the fact that many pollutants are transported with particles in the sewer systems and therefore are emitted into receiving waters. In this paper, significant emissions in commonly applied sewer systems (combined sewer system [CSS], separate sewer system [SSS]) are mentioned, and typical concentration ranges of SS are listed. Furthermore, data from selected pollutants (lead, polycyclic aromatic hydrocarbons [PAH]) are collected and concentration ranges in typical flow types of the sewer systems are calculated.

In the second part of the paper results from pollution load simulations are introduced. The calculations are based on assumptions from literature and were conducted in a hypothetical catchment. Main interest was the comparison of emissions out of SSS and CSS, shown for selected parameters SS, lead and PAH.

Results of the pollution load simulation show that emissions for surface runoff related compounds like lead are extremely high in the SSS in comparison to the CSS. This is mainly reasoned by the high elimination rate in the waste water treatment plant (WWTP). 
Abb. 1 Darstellung der Komponenten beider Kanalsysteme (Trennsystem/Mischsystem) und der wichtigsten Emissionen in Oberflächengewässer. MW-Kanal: Mischwasserkanal; SW-Kanal: Schmutzwasserkanal; $R W$-Kanal: Regenwasserkanal; $R W$ : Regenwetter; $T W$ : Trockenwetter; $K A$ : Kläranlage; $R K B$ : Regenklärbecken; grau: niederschlagsbedingte Einträge

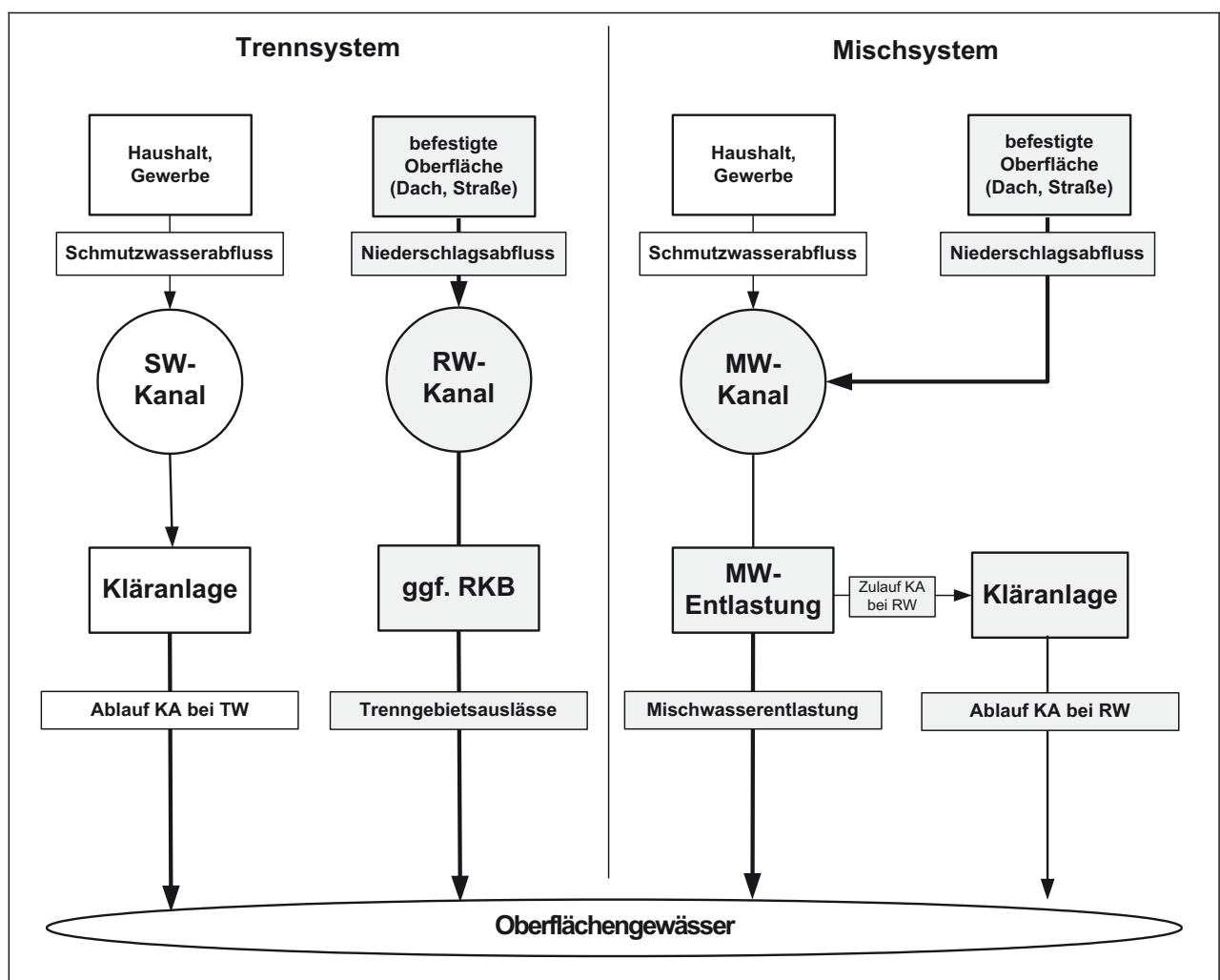

In summary, a general answer, which sewer system emits higher pollution loads is not possible. It is extremely dependent on the considered substance and its specific properties and resulting behaviour in the sewer systems. For a first estimation, new classes of substances are distinguished considering their origin (surface runoff versus dry weather flow) and their elimination capacity in the WWTP.

Keywords Pollutants $\cdot$ Pollution load simulations $\cdot$ Sewer systems $\cdot$ Suspended solids

\section{Einleitung}

Die Kanalsysteme sind innerhalb des urbanen Wasserkreislaufs wichtige Emissionsquellen für mögliche stoffliche Belastungen von Oberflächengewässern. Je nach vorhandenem Kanalsystem (Trenn- oder Mischsystem) sind verschiedene Einträge zu erwarten. Neben den Emissionen aus den Kläranlagen, die weitgehend konstant in ein Oberflächengewässer einleiten, sind zunehmend auch die niederschlagsbedingten Abflüsse (z.B. Mischwasserentlastungen) zu beachten. Diese treten je nach vorliegendem Kanalsystem und anderen Randbedingungen in höchst unterschiedlichen Formen auf (Abb. 1).

Im Trennsystem sind die niederschlagsbedingten Oberflächenabflüsse $\mathrm{zu}$ beachten, die teilweise hoch mit be- stimmten Schadstoffen belastet sein können (z.B. PAK, Schwermetalle) und häufig ohne jede Vorbehandlung als Trenngebietsauslässe in die Gewässer gelangen. Im Mischsystem können über die Mischwasserentlastungen sowohl aus dem Niederschlagsabfluss als auch aus dem Schmutzwasserabfluss Stoffe in die Gewässer eingetragen werden.

Aufgrund der potenziellen negativen Beeinträchtigungen (hydraulisch und stofflich) durch Einleitungen in die Oberflächengewässer sind gegenwärtig in Deutschland Anforderungen an die Behandlung der Emissionen aus der Siedlungsentwässerung in der fachlichen Diskussion.

Im Mischsystem wird vielfach die Einhaltung der Vorgaben des Arbeitsblattes 128 der ATV (jetzt DWA) als maßgebend angesehen (ATV 1992). Weitergehende Anforderungen, die sich beispielweise durch die Schutzbedürftigkeit des Gewässers ergeben, werden von der zuständigen Behörde zusätzlich im Einzelfall gefordert.

Im Trennsystem existieren bundesweit keine einheitlich geltenden Vorgaben. Häufig zum Einsatz kommen die Vorgaben des Merkblattes M 153 der DWA (ATV-DVWK 2007) oder wiederum einzelfallspezifische Regelungen. In Nordrhein-Westfalen gelten die Vorgaben des „Trennerlasses“" (MUNLV 2004).

Immissionsseitige Anforderungen für Niederschlagsabflüsse aus beiden Kanalsystemen, wie sie das BWK Merkblatt M 3 formuliert, werden in einzelnen Bundesländern (z. B. NRW) umgesetzt (BWK-M3 2004). 
Auf Bundesebene existieren gegenwärtig Überlegungen, sogenannte Mindestanforderungen bei Einleitung von Niederschlagsabflüssen in Grundwasser und Oberflächengewässer zu formulieren. Hierbei werden je nach Herkunftsfläche die Niederschlagsabflüsse in verschiedene Belastungsklassen aufgeteilt. Nur für die mäßig bis hoch belasteten Abflussarten muss dann eine Behandlung vorgesehen werden. Viele einzelne Beurteilungskriterien, beispielesweise auch der Feststoffparameter AFS, befinden sich derzeit noch in der Diskussion. Trotzdem ist zukünftig nicht auszuschließen, dass bundeseinheitliche Anforderungen an Niederschlagsabflüsse zur Einleitung in Oberflächengewässer zu beachten sind.

Bei den Stoffparametern fanden in den vergangenen Jahren neben den „klassischen“ Wasserparametern (z. B. CSB) zunehmend die organischen Schadstoffe und die von ihnen ausgehenden Wirkungen Beachtung in der Gewässerschutzdiskussion. Die Wasserrahmenrichtlinie (WRRL) trägt dieser Entwicklung Rechnung, indem sie neben biologischen Kenngrößen für den ökologischen Zustand auch einige stoffliche Kenngrößen nennt. Die im Anhang X der Richtlinie aufgeführte Liste der prioritären Stoffe (33 Stoffe bzw. Stoffgruppen) enthält überwiegend organische Verbindungen (z. B. PAK) (EU-Kommission 2001).

Viele dieser genannten Schadstoffe werden im urbanen Wasserkreislauf an Feststoffe assoziiert transportiert und werden daher mit diesen Feststoffen in die Oberflächengewässer eingetragen. Es ist daher wichtig, zukünftig vertiefende Erkenntnisse zum Aufkommen der Feststoffe und deren Kornverteilungen sowie verschiedener einzelner Schadstoffe in wichtigen Abflüssen der Kanalisation zu gewinnen.

Des Weiteren ist zu fragen, welche Kenngröße für die Beschreibung einer möglichen stofflichen Belastung der Gewässer maßgeblich ist. Grundsätzlich besteht die Möglichkeit, Vorgaben für mittlere bzw. maximale Konzentrationen oder emittierte Jahresfrachten festzulegen. Dem Aspekt der Stoffkonzentrationen in Niederschlagsemissionen wird in Abschn. 2 nachgegangen.

Eine zweite Möglichkeit, stoffliche Vorgaben festzuschreiben, besteht darin, zulässige emittierte Jahresfrachten festzulegen. Hierzu bietet die Schmutzfrachtsimulation, hier durchgeführt an einem fiktiven Einzugsgebiet, die Möglichkeit, einen Vergleich der Emissionen aus verschiedenen Kanalsystemen vorzunehmen (Abschn. 3).

\section{Aufkommensdaten (Konzentration) in wichtigen Abflüssen der Kanalisation}

\subsection{Feststoffe}

Die Gruppe der Feststoffe umfasst ungelöste Stoffe, die aus den verschiedensten Materialien, wie beispielsweise Tonmineralien oder Humussubstraten, bestehen können. Feststoffe spielen in Wassermatrizes eine bedeutende Rolle und werden aufgrund verschiedener Untersuchungsziele und mithilfe von variierenden Methoden bestimmt. In Deutschland werden sie häufig als ,abfiltrierbare Stoffe“ (AFS) erfasst. Auch bei Abflüssen in der Kanalisation wird den Feststoffen eine besondere Bedeutung beigemessen. Die mitgeführten Feststoffe können entweder als Problemstoff selbst (Verklebung des Interstitials) oder als Träger von zahlreichen Schadstoffen wirken. Die sich dabei potenziell einstellende Wirkung ist auch abhängig von gewässerseitigen Parametern, z.B. der Vorbelastung des Gewässers. Die Auswirkungen von feststoffreichen Mischwassereinleitungen auf ausgewählte Gewässerorganismen wurden von Borchardt (1992) in einem Fließrinnensystem untersucht. Selbst hohe Feststoffkonzentrationen von mehreren $100 \mathrm{mg} / 1$ hatten hinsichtlich der Drift und Mortalität keinen Effekt auf den Gewässerorganismus Gammarus pulex. Der Autor geht deshalb von überwiegend indirekten Wirkungen (s. o.) von Feststoffen in Fließgewässern aus. Allgemeingültige Fracht- oder Konzentrationsgrenzwerte für die AFS-Belastung in oberirdischen Gewässern sind daher nach derzeitigem Kenntnisstand nicht ableitbar (BWK-M3 2004).

Die in Niederschlagsabflüssen enthaltenen Feststoffe stammen aus dem Potenzial, das bereits auf der Fläche deponiert ist, z.B. Bodenoberflächenverunreinigungen wie Abfälle, Abrieb, mineralische Partikel und organische Feststoffe wie Laub, sowie durch die Feststoffbelastung des Niederschlags selbst. Die gemessenen AFS-Konzentrationen schwanken in einem großen Bereich. Die wesentlichen Faktoren beeinflussen, analog zu vielen anderen niederschlagsbedingten Schmutzstoffen,

- das Niederschlags-/Abflussgeschehen (Regenhöhe und Intensitätsverteilung, Vorbelastung u. a.),

- die luftseitige Grundbelastung (Staubbelastung Ballungsgebiet versus ländliches Gebiet),

- die Intensität der Straßenreinigung/Windverfrachtung und

- die Untersuchungsmethodik (Probennahme, Analyseverfahren u. a.).

Niederschlagsabflüsse von Oberflächen haben häufig höhere mineralische Anteile als Mischwasserentlastungen. Ferner wurde in einer Untersuchung von Geiger und Kutzner (2001) gezeigt, dass die Feststoffe in Straßenabflüssen und Trenngebietsabflüssen geringfügig besser absetzbar sind als in Mischwasserabflüssen.

Im Unterschied zu den Nährstoffen, die überwiegend schmutzwasserbürtig sind, tritt beim Parameter AFS im Mittel keine signifikante Verdünnung des Mischwasserabflusses durch die Oberflächenabflüsse auf. Trenngebietsabflüsse können also die gleichen AFS-Konzentrationen wie Mischwasserentlastungen aufweisen.

Gerade in Mischwasserkanälen treten zudem remobilisierbare Kanalablagerungen auf, die beim nächsten Regen- 


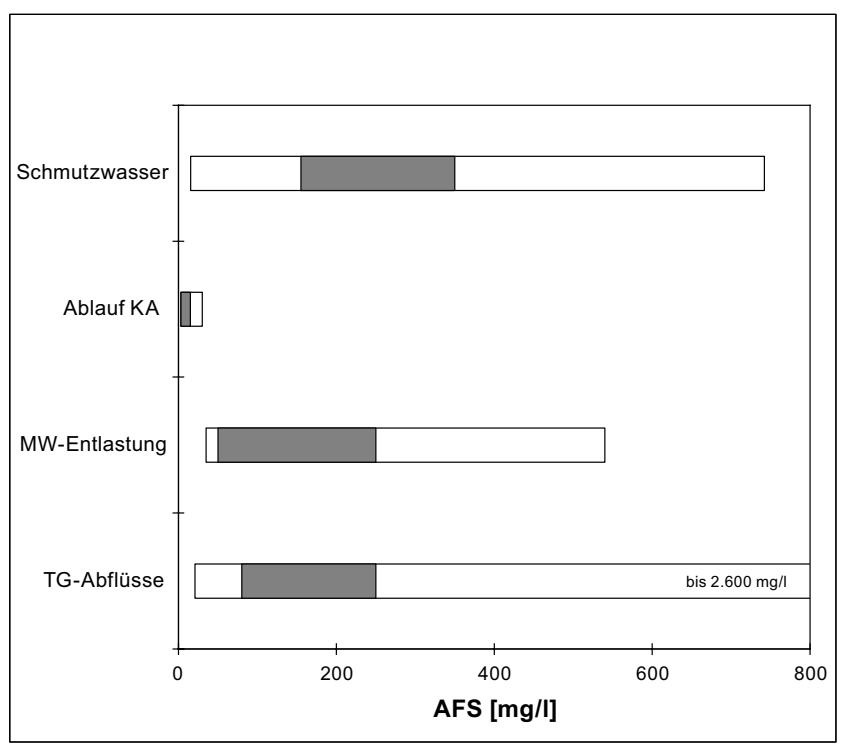

Abb. 2 Aufkommen von AFS in verschiedenen Abflussarten des Abwassersystems (Welker 2004); TG-Abflüsse: Trenngebietsabflüsse; weiße Balken: Min-Max-Werte; graue Balken: häufig vorkommende Werte

ereignis zu einer zusätzlichen AFS-Fracht im Mischwasserabfluss führen können. Zusammen mit der abgetragenen Sielhaut wird dieser Anteil als Kanalpotenzial bezeichnet (de Vries 1993). Das Kanalpotenzial kann einen wesentlichen Anteil an der abgeflossenen AFS-Menge eines Mischwassereignisses haben (Schulz 1995). Das Ablagerungsverhalten von Feststoffen im Mischwasserkanal ist abhängig vom Sammlerprofil, dem Gefälle sowie der Art und Größe des Trockenwetterabflusses. Eine allgemeingültige Beschreibung der Bedeutung des Kanalpotenzials ist aber nach Schulz (1995) nicht möglich.

Im Sinne der präventiven Rückhaltung von Feststoffen vor der Einleitung in ein Gewässer (s. o.) sollen Bauwerke zur Regenentlastung grundsätzlich so gestaltet werden, dass ein Rückhalt von Feststoffen begünstigt wird.

Dieses Ziel kann in der Mischwasserbehandlung konventionell durch Absetzbecken (z. B. Durchlaufbecken) erreicht werden. Bei weitergehenden Anforderungen an den Feststoffrückhalt kommen auch andere Verfahren (z.B. Wirbelabscheider, Bodenfilter) zum Einsatz. Trotz dieser Maßnahmen finden sich in Mischwasserüberläufen noch signifikante AFS-Gehalte (Abb. 2).

\subsection{Ausgewählte Schadstoffe}

In diesem Abschnitt werden ausgewählte, vorwiegend mit Feststoffen transportierte, Schadstoffe wie das Schwermetall Blei und die organische Schadstoffgruppe der PAK genauer betrachtet.

\subsubsection{Schwermetall Blei}

Wesentliche Ursachen für mögliche Belastungen durch das Schwermetall Blei in den Abflüssen der Kanalsysteme sind die Atmosphäre, aber auch Emissionen aus verschiedenen Materialien (Baumaterialien). Nach einer bundesweiten Bilanz stellen die niederschlagsbedingten Abflüsse (Mischwasserentlastung, Trenngebietsauslässe) im Vergleich zu den Kläranlagenabläufen einen bedeutenden Anteil an der Gewässerbelastung (Böhm et al. 2001).

Bezogen auf die gesamte Feststoffphase zeigt sich, dass ein großer Anteil der Schwermetalle an der feinpartikulären und nicht absetzbaren Fraktion $(<6 \mu \mathrm{m})$ anhaftet. $\mathrm{Zu}-$ sammen mit der Fraktion zwischen 6-60 $\mu \mathrm{m}$ bestimmen diese Fraktionen in vielen Fällen den Schwermetalltransport in Niederschlagsabflüssen (Sansalone und Buchberger 1997).

Untersuchungen zu Wirkungen von Schwermetalleinträgen aus niederschlagsbedingten Einträgen (z.B. Mischwasserentlastungen) sind bislang selten zu finden. Seidl et al. (1998) versuchten, mit der möglichen Hemmung der Sauerstoffproduktionsrate durch Schwermetalle, die Auswirkungen auf das Phytoplankton eines Gewässers nachzuweisen. Die ermittelten $\mathrm{EC}_{50}$-Konzentrationen (EC: effect concentration) liegen in diesem Testsystem für Zink bei ca. $340 \mu \mathrm{g} / \mathrm{l}$. Auch bei Kupfer und Blei werden, im Vergleich zu den Kontrollansätzen, deutliche Hemmwirkungen festgestellt. Die Autoren betonen, dass das wichtigste Kriterium für die Beurteilung der Wirkung von Schwermetallen die Verfügbarkeit der freien Elementformen ist.

Die Aufkommensdaten von Blei zeigen, dass im Vergleich zu den Abläufen der Kläranlagen signifikante Konzentrationen sowohl in Mischwasserentlastungen als auch in Trenngebietsabflüssen vorkommen können (Abb. 3). Insgesamt wird aber eine zurückgehende Bleibelastung prognostiziert, verursacht durch immissionsseitige Vorgaben (z.B. TA Luft) und die Substitution bleihaltiger Produkte (z. B. Benzin).

\subsubsection{Polyzyklische aromatische Kohlenwasserstoffe (PAK)}

Ebenfalls überwiegend von Niederschlagsabflüssen stammen die polyzyklischen aromatischen Kohlenwasserstoffe (PAK). Die PAK gelten als toxikologisch äußerst relevante Substanzgruppe. Insbesondere das Benzo[a]pyren wird als Leitsubstanz genannt. Bei dieser Verbindung muss von reproduktionstoxischen und mutagenen Wirkungen am Menschen ausgegangen werden. Deshalb wird die Relevanz für viele Umweltkompartimente sowohl für die Gruppe der PAK als auch für Benzo[a]pyren als hoch eingestuft (Abb. 4).

Obwohl die PAK als Stoffgruppe gemessen und bewertet werden, muss beachtet werden, dass jede Einzelverbindung spezifische Stoffeigenschaften, Wirkungen und Verteilungs- 


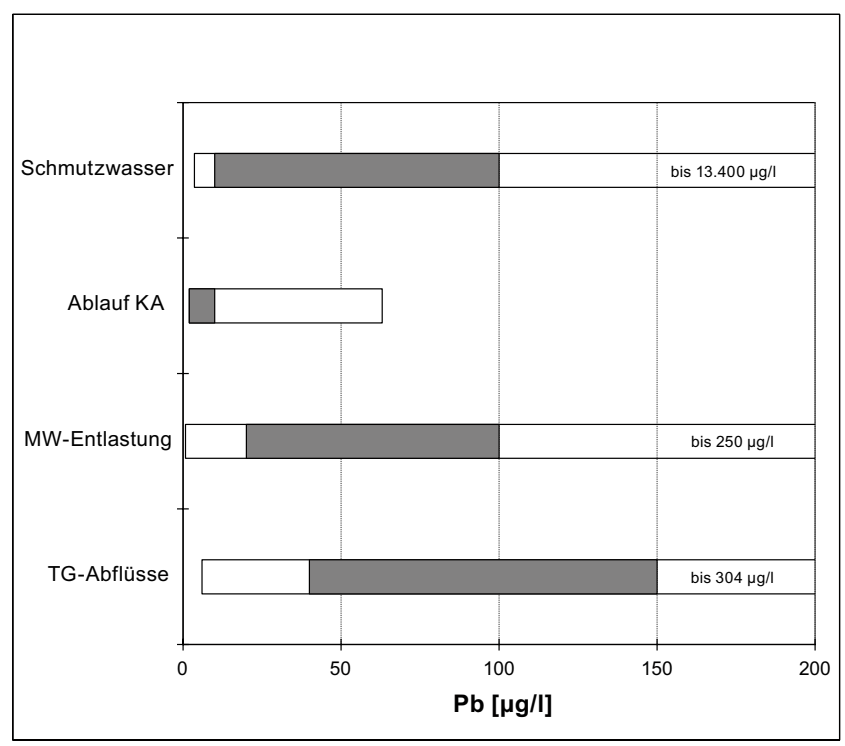

Abb. 3 Aufkommen von Blei in verschiedenen Abflussarten der Kanalsysteme des Abwassersystems (Welker 2004); TG-Abflüsse: Trenngebietsabflüsse; weiße Balken: Min-Max-Werte; graue Balken: häufig vorkommende Werte

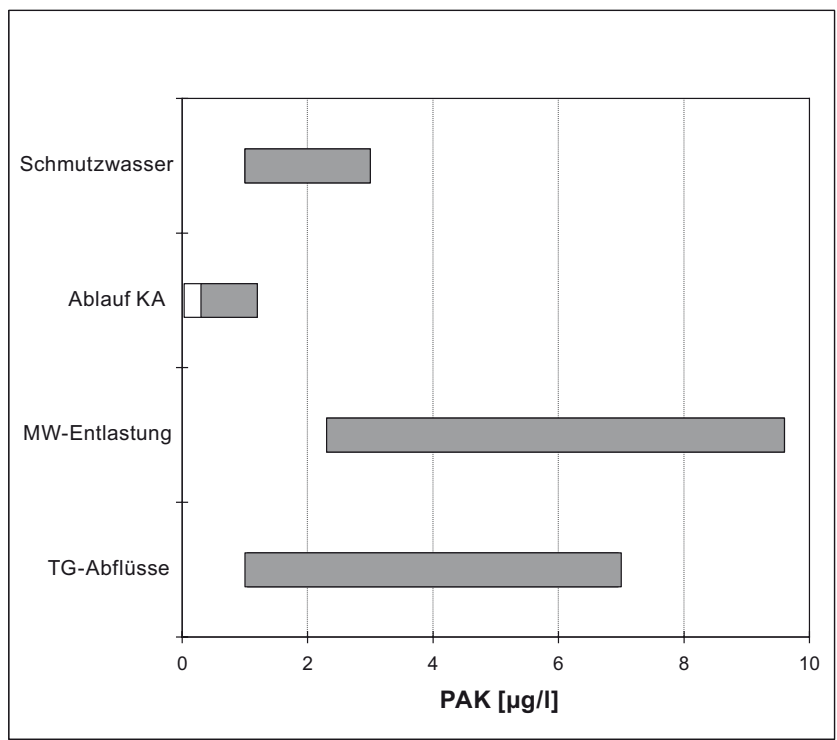

Abb. 4 Aufkommen von PAK in verschiedenen Abflussarten der Kanalsysteme des Abwassersystems (Welker 2004); TG-Abflüsse: Trenngebietsabflüsse; weiße Balken: Min-Max-Werte; graue Balken: häufig vorkommende Werte

muster in Umweltmedien aufweist. Die Hauptquellen, bezogen auf die Summe der PAK, sind Kleinfeuerungsanlagen, Großfeuerungsanlagen sowie Industrie- (Stahl) und Verkehrsemissionen (Haritopoulou 1996).

Der partikuläre Transport ist bei den Niederschlagsabflüssen vorherrschend; das gilt vor allem für die höher molekularen Verbindungen. Für die PAK kann daher insgesamt von einem überwiegend an Partikeln gebundenen Transport ausgegangen werden.

Im Mischsystem können Niederschlagsabflüsse zusammen mit dem Schmutzwasser durch die Mischwasserentlastungen bedeutend für den Eintrag in Gewässer sein. Hierzu sind bislang äußerst wenige Messungen durchgeführt worden. In Trennsystemen und den möglichen Regenwasserkanalabflüssen, ggf. nach Behandlung in Regenklärbecken, liegen ebenfalls sehr wenige Untersuchungen vor (Haritopoulou 1996).

Ein allgemeines Problem beim Datenvergleich von Messprogrammen ist, dass häufig verschiedene Bestimmungsmethoden angewandt und unterschiedliche Einzelverbindungen berücksichtigt wurden. Des Weiteren sind die Bedingungen der Ergebnisauswertung nicht immer klar dokumentiert. So ist bei der Mittelwertbildung nicht immer eindeutig, ob ausschließlich positive Proben berücksichtigt werden oder Bestimmungsgrenzen von nichtpositiven Proben einbezogen wurden. Diese Umstände werden häufig bei Vergleichsbetrachtungen außer Acht gelassen und erschweren eine umfassende Bewertung von Messergebnissen.

\section{Emittierte Jahresfrachten - Ergebnisse der Schmutzfrachtsimulation}

Gegenstand dieses Abschnitts sind Simulationen und Berechnungen zur Verteilung von Stoffen in Kanalsystemen. Es werden drei Beispielstoffe (AFS, Blei, PAK) ausgewählt, welche die Feststoffe selbst oder vorwiegend an Feststoffen sorbierte Verbindungen abbilden. Die Ausführungen erstrecken sich zunächst auf ein nahezu $100 \%$-iges Mischsystem, das Modellnetz aus dem ATV-Arbeitsblatt A128, mit den Emissionen Mischwasserentlastung und Ablauf der Kläranlage. Anschließend werden - unter Verwendung der gleichen stofflichen Eingangsdaten - die Emissionen für ein Trennsystem berechnet, die aus den Trenngebietsauslässen und den Abläufen der Kläranlage in das Gewässer gelangen.

\subsection{Methodik}

Als Einzugsgebiet wird ein fiktives Gebiet verwendet, das als Beispielgebiet (9900 Einwohner) im Arbeitsblatt der ATV-A 128 aufgeführt ist (ATV 1992). Es wird das Modell KOSMO (KOntinuierliche Schmutzfracht MOdellierung) verwendet, das $\mathrm{zu}$ den hydrologisch-hydrodynamisch-deterministischen Modellen gehört (Schmitt 1994). Die Stoffkonzentration des Oberflächenabflusses wird in dieser Arbeit mit dem Ansatz „Akkumulation/Abtrag“ berechnet. In den Regenentlastungsbauwerken werden die Stofffrachten entsprechend den Abflussverhältnissen aufgeteilt. Die Regenbelastung ist bei allen Varianten konstant. Es wird eine 
Abb. 5 Emittierte Jahresfrachten (Vergleich MischsystemTrennsystem) für AFS, Blei $(\mathrm{Pb}), \mathrm{PAK} ; M S$ : Mischsystem; TS: Trennsystem; OA TS: Oberflächenabfluss Trennsystem, entspricht Trenngebietsauslässe; RE MS: Mischwasserentlastung

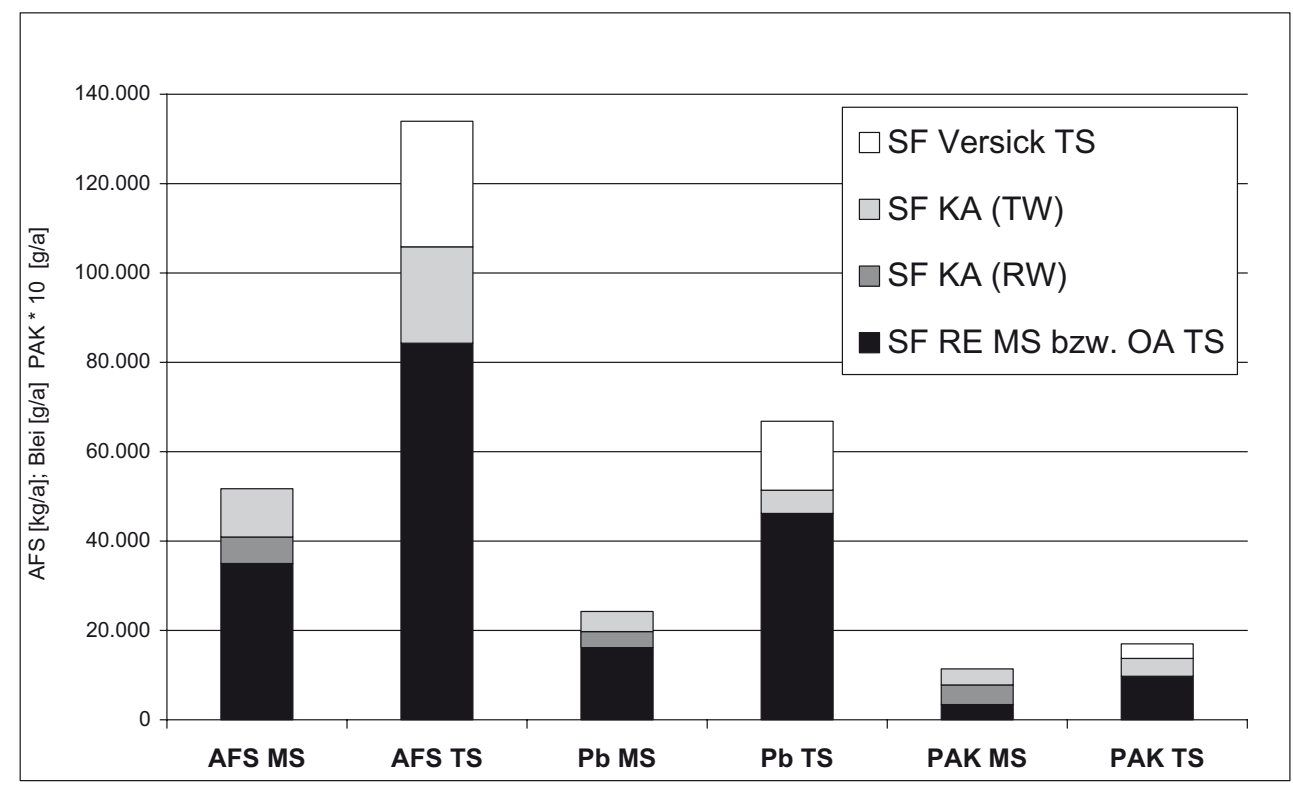

einjährige Regenreihe aus dem südwestdeutschen Raum mit einer Gesamtniederschlagsmenge von $825 \mathrm{~mm}$ verwendet.

Für die Berechnungen werden festgelegte Stoffkonzentrationen für die Abflüsse Trockenwetterabfluss, Oberflächenabfluss und Kläranlagenablauf (unterschieden nach Trocken- und Regenwetter) verwendet. Die Kriterien zur Festlegung der Konzentrationen und weitere Details sind bei Welker $(2007 a, b)$ nachzulesen.

\subsection{Ergebnisse (Vergleich Misch- und Trennsystem)}

Als Beurteilungsgrößen werden die emittierten Jahresfrachten (z. B. kg/a) aus dem Mischsystem (Mischwasserwasserentlastung und Ablauf der Kläranlage) im Vergleich zu den Emissionen des Trennsystems (Trenngebietsauslässe und Ablauf der Kläranlage) herangezogen.

In Trennsystemen wird häufig ein Teil der Niederschlagsabflüsse der Versickerung zugeführt und gelangt damit nicht direkt in die Fließgewässer. Dieser Anteil ist vom Einzelfall abhängig und extrem unterschiedlich. In diesem Vergleich wird er mit $25 \%$ des jährlich abfließenden Niederschlagsabflusses angesetzt. Die hieraus berechnete Versickerungsfracht wird nicht pauschal abgezogen, sondern in der Grafik als weißer Balken auf die Summe aus Kläranlagenablauffracht (grauer Balken) und verbleibender Niederschlagsabflussfracht (schwarzer Balken) aufgesetzt (Abb. 5 bei $\mathrm{Pb}$ TS). Dadurch wird die Darstellung einer Spannweite des Versickerungsfrachtanteils ermöglicht.

Die Einzelfrachten der Kläranlagenabläufe bei Regenwetter und bei Trockenwetter beim Mischsystem werden ebenfalls mit unterschiedlichen Einfärbungen (Regenwetter: schraffierter Balken; Trockenwetter: grauer Balken) dargestellt. Komplettiert wird der Gesamtfrachtbalken im
Mischsystem durch die Darstellung der Einzelstofffracht der Mischwasserentlastung (schwarzer Balken) (Abb. 5).

Es ist zu erkennen, dass bei allen oberflächenbürtigen Stoffen, beispielsweise dem Blei, die emittierte Gesamtfracht im Trennsystem - auch nach dem Abzug der Versickerungsfracht - wesentlich höher ist als die im Mischsystem. Dies ist durch die Mitbehandlung belasteter Niederschlagsabflüsse in Kläranlagen im Mischsystem begründet.

Durch gute Rückhalteleistungen in der Kläranlage werden beispielsweise die Schwermetalle in den Klärschlamm verfrachtet; hierdurch gelangen geringere Mengen in den Ablauf und damit in die Fließgewässer. Am Beispiel der Schwermetalle wird aber auch ersichtlich, dass durch die Einführung der Versickerung lediglich eine Verschiebung von Stoffströmen stattfindet. Beim Übergang vom Trennin das Mischsystem werden Schwermetalle verstärkt vom Fließgewässer in den Klärschlamm verlagert. Bei einer anschließenden landwirtschaftlichen Verwertung des Klärschlammes würde diese Stofffracht also in das BodenGrundwasser-System verlagert.

Insgesamt können durch den Vergleich eines Mischsystems und eines Trennsystems in einem fiktiven Beispieleinzugsgebiet Tendenzen zum Aufkommen und zur Verteilung herkömmlicher und möglicherweise in Zukunft bedeutsamer Stoffe mit analogen Stoffeigenschaften aufgezeigt werden.

\section{Fazit}

Die Ergebnisse der Literaturauswertung zur Beschaffenheit von Abflüssen in Kanalsystemen lassen erkennen, wie groß die Vielfalt der untersuchten Verbindungen mit ihren variierenden Kenngrößen, Stoffeigenschaften und Wirkungen ist. 
Als charakteristisches Unterscheidungskriterium für eine erste Einteilung der Substanzen wird die Herkunft der Stoffe definiert, die eindeutig die Belastung der verschiedenen Matrizes des Abwassersystems bestimmt. Während sich die aus dem Schmutzwasser stammenden (,schmutzwasserbürtigen") Verbindungen (z.B. Humanarzneimittel) überwiegend im Kläranlagenablauf finden, sind die von der Oberfläche stammenden („oberflächenbürtigen“) Verbindungen (z. B. Blei) vor allem in den niederschlagsbedingten Abflüssen zu erwarten. Weitere Faktoren, die das Aufkommen und die Verteilung von Stoffen im Abwassersystem beeinflussen, sind der Anteil an partikulärem Transport sowie die Rückhalteleistung in der Kläranlage.

Allgemein lässt sich folgern, dass emittierte Niederschlagsabflüsse im Vergleich zu anderen Emissionsquellen (z.B. Abläufe der Kläranlagen) in erheblichem Maße zur Gewässerbelastung beitragen können.

Bei der Zusammenstellung von Aufkommensdaten zur Beschaffenheit von Niederschlagsabflüssen ist das hohe Datendefizit hervorzuheben, insbesondere bei den organischen Schadstoffen (z. B. PAK), in den Komponenten Mischwasserabfluss bzw. Mischwasserentlastungen und den Trenngebietsabflüssen. Damit ergibt sich zukünftig die dringende Notwendigkeit zu weiteren Messungen.

Hierbei ist eine enge Kooperation zwischen Fachleuten der Siedlungswasserwirtschaft, insbesondere mit Erfahrungen mit Messprogrammen auf Regenwasserbehandlungsanlagen und Experten der chemischen Spurenanalytik mit Erfahrungen mit schwierigen Wassermatrizes notwendig. Als Ergänzung sind Simulationsberechnungen hilfreich, die Tendenzaussagen zum Aufkommen und der Verteilung von neuen Stoffen im jeweiligen Einzugsgebiet ermöglichen. Es können Belastungsschwerpunkte aufgezeigt werden, die wertvolle Hinweise für die Konzeption der Messprogramme liefern.

Neben den Defiziten bei den Aufkommensdaten besteht weiterhin die Notwendigkeit zur Definition von Anforderungen, die bei Einleitung von niederschlagsbedingten Abflüssen in die Gewässer zu beachten sind. Hierbei könnten erste Worst-Case-Betrachtungen mit maximalen Annahmen zum Ausmaß der Belastung und zur Empfindlichkeit eines Gewässers für die Abschätzung der Auswirkungen von Stoffkonzentrationen in Gewässern eine Entscheidungsgrundlage liefern.

\section{Literatur}

ATV (Abwassertechnische Vereinigung e.V.) (1992) Richtlinien für die Bemessung und Gestaltung von Regenentlastungsanlagen in
Mischwasserkanälen (A 128). GFA - Gesellschaft zur Förderung der Abwassertechnik e. V., Hennef

ATV-DVWK (2007) Merkblatt ATV-DVWK M 153 (August), Handlungsempfehlungen zum Umgang mit Regenwasser. Hennef

Böhm E, Hillenbrand T, Marscheider-Weidemann F, Schempp C, Fuchs S, Scherer U (2001) Bilanzierung des Eintrags prioritärer Schwermetalle in Gewässer. UBA-Texte 29/01, Forschungsbericht 29822 243, Umweltbundesamt Berlin

Borchardt D (1992) Wirkungen stoßartiger Belastungen auf ausgewählte Fließgewässerorganismen - ein Beitrag zur Beurteilung ökologischer Schäden durch Niederschlagwassereinleitungen aus Kanalisationen. Schriftenreihe des Fachgebietes Siedlungswasserwirtschaft Universität Kassel, Bd 10. Gesamthochschule Kassel

BWK-M3 (2004) Merkblatt zur Ableitung von immissionsorientierten Anforderungen an Misch- und Niederschlagswassereinleitungen unter Berücksichtung örtlicher Verhältnisse, 2. Aufl. BWK (Bund der Ingenieure für Wasserwirtschaft, Abfallwirtschaft und Kulturbau e. V.), Düsseldorf

EU Kommission (2001) Vorschlag für eine Entscheidung des Europäischen Parlaments und des Rates zur Festlegung der Liste der prioritären Stoffe im Bereich der Wasserpolitik. EU Kommission, Brüssel

Geiger WF, Kutzner R (2001) Neuere Entwicklungen im Bereich der Misch- und Regenwasserbehandlung. ATV-DVWK-Seminar „Aktuelles und Bewährtes zur Regenwasserbehandlung“, Fulda, 2001, ATV-DVWK, Hennef, S 1-20

Haritopoulou T (1996) Polyzyklische aromatische Kohlenwasserstoffe und Schwermetalle in urbanen Entwässerungssystemen - Aufkommen, Transport und Verbleib. Schriftenreihe des Instituts für Siedlungswasserwirtschaft, Bd 77, Universität Karlsruhe (TH). Oldenbourg, München

MUNLV - Ministerium für Umwelt des Landes Nordrhein-Westfalen (2004) Anforderungen an die Niederschlagsentwässerung im Trennverfahren, RdErl, 25.5.2004

Sansalone J, Buchberger SG (1997) Characterisation of solid and metal element distribution in urban highway storm water. Water Sci Technol 36(8-9):155-160

Seidl M, Huang V, Mouchel JM (1998) Toxicity of combined sewer overflow on river phytoplankton: the role of heavy metals. Environ Pollut 101:107-116

Schulz O (1995) Gebietsspezifische Schmutzfrachtpotenziale flach verlegter und vorentlasteter Mischwassernetze. Mitt LeichtweissInstitut Wasserbau Techn Universität Braunschweig 137:1-172

Schmitt TG (1994) Detaillierte Schmutzfrachtberechnung nach ATVArbeitsblatt A 128. Korresp Abwasser 40(12):2212-2230

de Vries JM (1993) Bestimmung des Schmutzfrachtpotentials in flach verlegten Misch- und Trennnetzen infolge abfließenden Niederschlagwassers. Mitteilungen des Leichtweiss-Institut Wasserbau Techn Universität Braunschweig 125:1-132

Welker A (2004) Schadstoffströme im urbanen Wasserkreislauf - Aufkommen und Verteilung, insbesondere in den Abwasserentsorgungssystemen. Habilitationsschrift, Schriftenreihe der TU Kaiserslautern, FG Siedlungswasserwirtschaft, Bd 20

Welker A (2007a) Assessment of organic pollutants with respect to occurrence and fate in combined sewer systems and possible impacts on receiving waters. Water Sci Technol 56(10):141-148

Welker A (2007b) First estimation of occurrence and fate of organic substances in combined sewer systems with pollution load simulations. Water Practice Technol 